\title{
Transverse-Coherence Properties of the Free-Electron-Laser FLASH at DESY
}

\author{
A. Singer,* I. A. Vartanyants, ${ }^{\dagger}$ M. Kuhlmann, S. Duesterer, R. Treusch, and J. Feldhaus \\ HASYLAB at DESY, Notkestrasse 85, D-22607 Hamburg, Germany
}

(Received 21 August 2008; published 18 December 2008)

\begin{abstract}
A general theoretical approach based on the decomposition of statistical fields into a sum of independently propagating transverse modes was used for the analysis of the coherence properties of the new free-electron laser source FLASH operated at $13.7 \mathrm{~nm}$ wavelength. The analysis shows that several transverse modes are contributing to the total radiation field of FLASH. The results of theoretical calculations are compared with measurements using Young's double-slit experiment. The coherence lengths in the horizontal and in the vertical directions $20 \mathrm{~m}$ downstream from the source are estimated at 300 and $250 \mu \mathrm{m}$, respectively.
\end{abstract}

DOI: 10.1103/PhysRevLett.101.254801

PACS numbers: 41.60.Cr, 42.25.Bs, 42.25.Kb

Coherence is one of the most prominent features of laser sources. With the advent of free-electron laser (FEL) based on the self amplified spontaneous emission (SASE) principle $[1,2]$ the possibility of building coherent sources with $\mathrm{x}$-ray wavelengths has become feasible. Several projects around the world are already under construction in the USA, Japan and Europe [3]. The first FEL for extended ultraviolet (XUV) wavelengths FLASH (Free-electron LASer in Hamburg) has started its user operation at DESY in 2005 [4]. The measurement of the coherence properties of existing FELs is of vital importance for understanding the physical principles that lie behind the SASE generation of coherent beams, for optimization of the parameters of these sources, and for the construction of beam lines. These measurements are also important for planning future experiments on these sources that exploit the coherence properties of high power FEL beams. These can be, for example, coherent diffraction experiments that use sufficiently sampled diffraction data for phase retrieval and reconstruction of the real space structure of a sample $[5,6]$. The first demonstration experiments of the possibility of single pulse coherent diffraction imaging were performed recently at FLASH $[7,8]$. In future when x-ray FELs, with a unique femtosecond time structure, will become available this approach can even be used for different applications in materials science [9] and biology including such exciting possibilities as single molecule imaging [10].

A variety of different approaches may be used to analyze the coherence properties of FELs. One of them is based on a detailed modelling of the SASE process by performing calculations of nonlinear electromagnetic equations at different conditions of operation (linear regime, saturation, etc.) [11]. Another possible approach is based on the use of results of statistical optics $[12,13]$, when the statistics of radiation fields are analyzed with very general assumptions about the origin of the radiators. In this Letter we choose this second approach to describe the coherence properties of the FEL beam originating from FLASH. In the same way as for conventional lasers, FELs based on the SASE principle can be described as a source with a finite number of transverse and longitudinal modes [1]. As we are interested in the coherence properties of the FLASH beam in the transverse direction, we use a decomposition of the statistical field into a sum of independently propagating transverse modes and show that for a source as coherent as FLASH only a few modes contribute substantially to the total radiation field.

Young's double-slit experiment [12] is one of the most efficient and widely used methods for measuring the transverse coherence properties of light beams [14], VUV and $\mathrm{x}$-ray radiation produced by synchrotron sources $[15,16]$, electron beams [17] and even matter waves [18]. In our experiments we have used the same approach and performed double-slit experiments to analyze the transverse coherence properties of FLASH.

The second order correlations of the wave fields are described in the theory of coherence by the mutual coherence function (MCF) $\Gamma_{12}(\tau)$, that defines the correlations between two complex values of the electric field $E\left(\mathbf{r}_{1}, t\right)$ and $E^{*}\left(\mathbf{r}_{2}, t+\tau\right)$ at different points $\mathbf{r}_{1}$ and $\mathbf{r}_{2}$ and separated by the time interval $\tau$

$$
\Gamma\left(\mathbf{r}_{1}, \mathbf{r}_{2}, \tau\right)=\left\langle E\left(\mathbf{r}_{1}, t\right) E^{*}\left(\mathbf{r}_{2}, t+\tau\right)\right\rangle,
$$

where the brackets \langle\rangle indicate the ensemble average. Correlations of the field in the spatial-frequency domain are determined by the cross-spectral density function (CSD) $W\left(\mathbf{r}_{1}, \mathbf{r}_{2}, \omega\right)$, which is a Fourier transform of the $\mathrm{MCF}$

$$
W\left(\mathbf{r}_{1}, \mathbf{r}_{2}, \omega\right)=\int_{-\infty}^{\infty} \Gamma_{12}(\tau) e^{-i \omega \tau} d \tau .
$$

The spectral density of the field $S(\mathbf{r}, \omega)$ is defined as the CSD function taken at the same position $S(\mathbf{r}, \omega)=$ $W(\mathbf{r}, \mathbf{r}, \omega)$. The normalized versions of the two functions (1) and (2) are the complex degree of coherence $\gamma\left(\mathbf{r}_{1}, \mathbf{r}_{2}, \tau\right)$ and the spectral degree of coherence $\mu\left(\mathbf{r}_{1}, \mathbf{r}_{2}, \omega\right)$, respectively. 
It has been shown [13], that under very general conditions, one can represent the CSD of a partially coherent statistically stationary field of any state of coherence as a sum of independent coherent modes [19] $W\left(\mathbf{r}_{1}, \mathbf{r}_{2}\right)=$ $\sum_{j=0} \beta_{j} \psi_{j}^{*}\left(\mathbf{r}_{1}\right) \psi_{j}\left(\mathbf{r}_{2}\right)$, where $\beta_{j}$ and $\psi_{j}(\mathbf{r})$ are the eigenvalues and eigenfunctions that satisfy the Fredholm integral equation $\int W\left(\mathbf{r}_{1}, \mathbf{r}_{2}\right) \psi_{j}\left(\mathbf{r}_{1}\right) d \mathbf{r}_{1}=\beta_{j} \psi_{j}\left(\mathbf{r}_{2}\right)$. For our purposes it is especially important to calculate correlation functions at different distances from the source. These values of the CSD can be obtained at different distances by propagation of the individual coherent modes. Because of statistical independence of the modes [13] the CSD after propagating a distance $z$ is given as a sum of propagated modes $\phi_{j}(\mathbf{r}, z)$ with the same eigenvalues $\beta_{j}$

$$
W\left(\mathbf{r}_{1}, \mathbf{r}_{2}, z\right)=\sum_{j=0} \beta_{j} \phi_{j}^{*}\left(\mathbf{r}_{1}, z\right) \phi_{j}\left(\mathbf{r}_{2}, z\right) .
$$

We apply this general approach to describe correlation properties of the wavefield originating from the FEL. We substitute a real FEL source by an equivalent model planar source described by a Gaussian Schell-model (GSM) with a CSD [13] $W\left(\mathbf{r}_{1}, \mathbf{r}_{2}\right)=A_{0}^{2} \exp \left[-\left(\mathbf{r}_{1}^{2}+\mathbf{r}_{2}^{2}\right) /\left(4 \sigma_{I}^{2}\right)-\right.$ $\left.\left(\mathbf{r}_{2}-\mathbf{r}_{1}\right)^{2} / 2 \sigma_{\mu}^{2}\right]$, where $\sigma_{I}$ and $\sigma_{\mu}$ are the values of the standard deviation of the spectral density and spectral degree of coherence at the source plane, coordinates $\mathbf{r}_{1}$ and $\mathbf{r}_{2}$ lie in the plane of the source and $A_{0}^{2}$ is the normalization constant. Coherent modes for the GSM source are well known [20,21] and can be decomposed for each transverse direction $\psi_{l, m}(x, y)=\psi_{l}(x) \psi_{m}(y)$ and $\beta_{l, m}=$ $\beta_{l} \beta_{m}$ [22]. The propagated modes $\phi_{j}(x, z)$ at distance $z$ from the source are described by the Gaussian Hermitemodes [23]. The eigenvalues $\beta_{j}$ have a power law dependence with the number of the modes $\beta_{j} / \beta_{0}=\kappa^{j}$, where $\kappa$ is a constant depending on the parameters of the GSM source [23]. Using these eigevalues and eigenfunctions in Eq. (3) the CSD and hence correlations of the fields in the coordinate-frequency domain can be calculated at any distance from the GSM source.

We used this approach to make a realistic and simple estimate of the coherence properties of the existing FLASH source. For calculations we take parameters of FLASH as reported in [4] at a fundamental wavelength of $13.7 \mathrm{~nm}$. Simulations were made for a GSM source with the value of the standard deviation $\sigma_{I}=68 \mu \mathrm{m}$. This corresponds to the FWHM of the source size of $160 \mu \mathrm{m}$ reported in Ref. [4]. The value of the standard deviation $\sigma_{\mu}=62 \mu \mathrm{m}$ was obtained by fitting using Eq. (3) to match the values of the FWHM of the beam size, $2.1 \mathrm{~mm}$, and the angular divergence of $90 \mu \mathrm{rad}$ measured $23.5 \mathrm{~m}$ downstream from the source [4]. Seven modes were used in (3) for that calculation. When parameters $\sigma_{I}$ and $\sigma_{\mu}$ of the GSM source were defined the CSD was calculated at a distance $20 \mathrm{~m}$ downstream from the source using Eq. (3). In Fig. 1 results of these calculations are presented. An analysis of the obtained results shows that for the parameters of FLASH a small number of transverse modes contribute
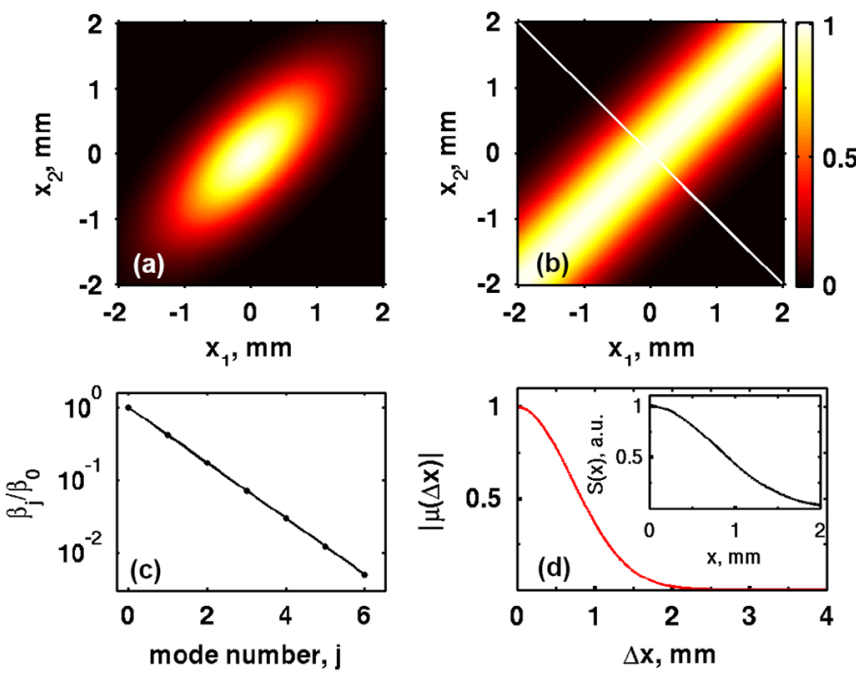

FIG. 1 (color online). (a) Modulus of the cross-spectral density $\left|W\left(x_{1}, x_{2}\right)\right|$. (b) Modulus of the spectral degree of coherence $\left|\mu\left(x_{1}, x_{2}\right)\right|$. (c) The ratio $\beta_{j} / \beta_{0}$ of the eigenvalue $\beta_{j}$ to the lowest order eigenvalue $\beta_{0}$ as a function of mode number $j$. (d) Modulus of the spectral degree of transverse coherence $|\mu(\Delta x)|$ taken along the white line in (b). In the inset spectral density $S(x)$ is shown. Calculations are made in the frame of a Gaussian Schell-model source $20 \mathrm{~m}$ downstream from the source with the wavelength $\lambda=13.7 \mathrm{~nm}$, when the FEL operates at its saturation.

to the total field [Fig. 1(c)]. Parameter $\kappa=0.414$ in these conditions that means that the contribution of the second mode is about $40 \%$ of the fundamental, and the contribution of the sixth mode is more then 2 orders of magnitude smaller than the fundamental $\beta_{6} / \beta_{0}=\kappa^{6} \approx 5 \times 10^{-3}$. As a consequence this demonstrates that our model source is not fully coherent. Using obtained values of the CSD we also calculated the values of the spectral density $S(\mathbf{r})$ and the modulus of the spectral degree of coherence $\mid \mu\left(x_{2}-\right.$ $\left.x_{1}\right) \mid$ for different separations between the points $x_{1}$ and $x_{2}$ [Fig. 1(d)]. As our source is described as a Gaussian source these functions can be described are Gaussian as well at a distance $20 \mathrm{~m}$ from the source with $\sigma_{I}(z)=764 \mu \mathrm{m}$ and $\sigma_{\mu}(z)=715 \mu \mathrm{m}$.

The transverse coherence was measured using a doubleslit experiment at a fundamental wavelength of $13.7 \mathrm{~nm}$ during the commissioning phase of FLASH. The average energy per pulse during the experiment was about $10 \mu \mathrm{J}$ which is well below the saturation that was reached at $40 \mu \mathrm{J}$ in an earlier experiment [4]. At these average energies about $10^{12}$ photons are produced per pulse. FLASH was operated at $1 \mathrm{MHz}$ repetition rate with 10 bunches per train and a $5 \mathrm{~Hz}$ repetition rate of trains. Because of the lower average energy the apparent source size during this experiment was about 2.5 times bigger than reported in [4] when measurements were done at saturation [24].

The experiment was performed with a set of horizontal and vertical slits laser cut into an $80 \mu \mathrm{m}$ thick stainless steel foil that was positioned at a distance $z_{1}=20 \mathrm{~m}$ 
downstream from the last operating undulator module in the FEL tunnel. The distance $d$ between the slit centers was 150,300 , and $600 \mu \mathrm{m}$ for both vertical and horizontal pairs. The individual slit width was $a=30 \mu \mathrm{m}$ for the first two pairs and $a=50 \mu \mathrm{m}$ for the last pair. The detection scheme consists of a fluorescent screen (Ce doped YAG crystal) converting the XUV radiation into the visible range. The resulting fluorescent radiation was imaged with a standard objective lens with a focal length $f=50 \mathrm{~mm}$ onto a Basler A311f CCD camera. In the geometry of the experiment the effective detector pixel size was $29.4 \mu \mathrm{m}$ in the vertical direction and $20.8 \mu \mathrm{m}$ in the horizontal. The detector was located at a distance $z_{2}=4.438 \pm 0.005 \mathrm{~m}$ downstream from the slit mask. Each interference pattern measured in this experiment was a result of the accumulation of ten bunches of a single train of FEL radiation. Each measurement for a given slit separation was repeated 10 times.

The typical interference pattern measured on the detector with a vertical slit separation of $150 \mu \mathrm{m}$ is shown in Fig. 2(a). One can see that interference fringes are very well resolved on the detector. From these 2D data sets 1D interference patterns were obtained by choosing an area on the detector centered at the maximum intensity and averaging over three pixels [shown in Fig. 2(a)] in the direction perpendicular to fringes. The averaging procedure was necessary to increase the statistics of the measured signal.

The interference pattern $I(P)$ measured at the point $P$ of the detector for the narrow band radiation [26] can be well
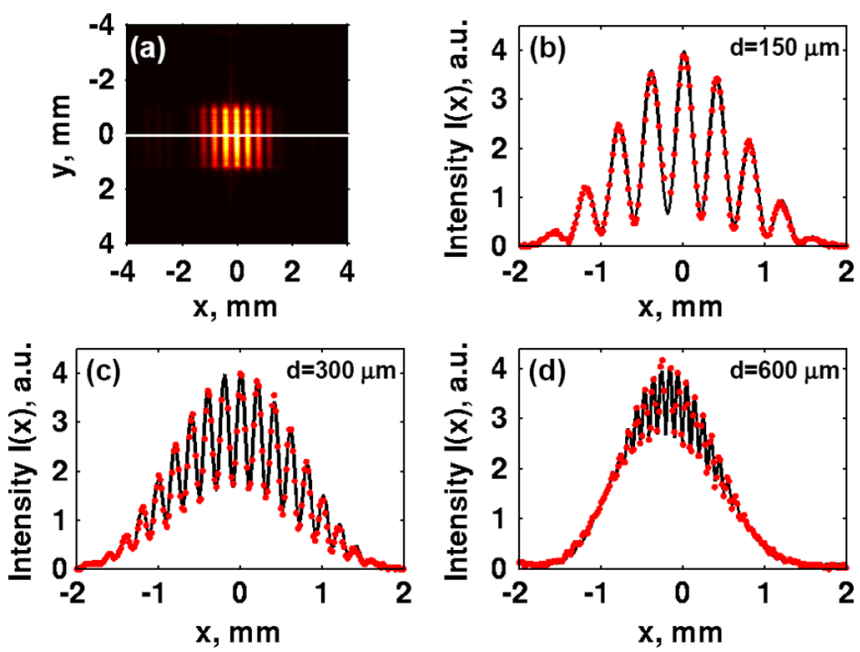

FIG. 2 (color online). Results of a double-slit experiment. (a) A typical data set measured on detector with the vertical slits of $150 \mu \mathrm{m}$ separation. Each image is a result of accumulation of ten bunches. The framed region in the image of three pixels wide was used for further analysis. (b)-(d) Results of the fit (solid lines) to experimental data (points) for different slit separation $d=150 \mu \mathrm{m}(\mathrm{b}), d=300 \mu \mathrm{m}(\mathrm{c}), d=600 \mu \mathrm{m}(\mathrm{d})$ in the horizontal direction. The error bars of the data are less than $3 \%$ and are smaller than the symbols used to represent the data. The error metric defined as $E=\sum_{i=1}^{N}\left(I_{i}^{\exp }-I_{i}^{\mathrm{fit}}\right)^{2} / \sum_{i=1}^{N}\left(I_{i}^{\mathrm{fit}}\right)^{2}$ was less than 0.002 for all fits. described by the following expression [12]

$$
\begin{aligned}
I(P)= & I_{1}(P)+I_{2}(P)+2 \sqrt{I_{1}(P) I_{2}(P)}\left|\gamma_{12}(\tau)\right| \\
& \times \cos \left[\omega \tau-\alpha_{12}(\tau)\right],
\end{aligned}
$$

where $I_{1}(P)$ and $I_{2}(P)$ are intensities at $P$ corresponding to propagation of radiation from each of the slits separately, $\tau$ is the time delay for the radiation to reach point $P$ at the detector from slits one and two and $\alpha_{12}(\tau)$ is the relative phase. For narrow bandwidth light, the complex degree of coherence can be approximated by $\gamma_{12}(\tau) \approx \gamma_{12}(0) \times$ $\exp (-i \omega \tau)$ leading to a relationship $\left|\gamma_{12}(0)\right|=\left|\mu_{12}(\omega)\right|$. Hence we can directly compare results of the double-slit experiment with the results obtained from the mode representation of GSM source. The periodicity of the fringes in the double-slit experiment is determined by the phase factor $\delta(P)=\omega \tau(P)$. In the paraxial approximation this phase factor is given by $\delta(x)=2 \pi d\left(x-x_{0}\right) /\left(\lambda z_{2}\right)$, where $x$ is the coordinate in direction perpendicular to interference fringes and $x_{0}$ is the center position of the inteference pattern. Because of the small slit widths and a long distance to the detector the far-field conditions are well satisfied and the Fraunhofer limit for the diffraction pattern of individual slits can be used with a good approximation.

Expression (4) was used for fitting all the series of experimental data. Typical results of the fit for different slit separations are shown in Figs. 2(b)-2(d). One can see from these results that the experimental data are very well reproduced by the theoretical fit. As a result of the fitting the absolute value of the complex coherence factor $\left|\gamma_{12}\right|$ was obtained for each slit separation for both the horizontal and vertical directions (see Fig. 3). To compare the obtained values of the complex coherence factor with the intensity distribution we plotted on the same figure the intensity distribution obtained by rescaling the experimen-

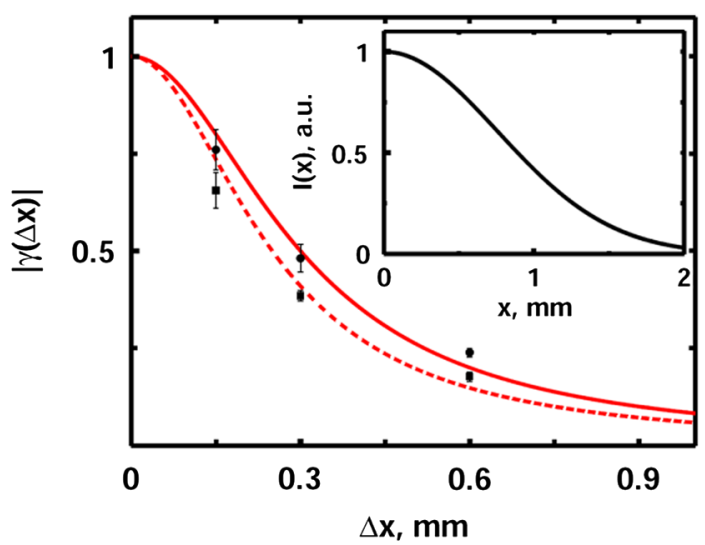

FIG. 3 (color online). Modulus of the complex degree of coherence as function of slit separation in the horizontal (circles) and vertical (squares) directions obtained as a result of the fit to experimental data. Lorentzian fit to obtained values of $|\gamma(\Delta x)|$ is shown by solid (horizontal direction) and dashed (vertical direction) lines. The intensity distribution scaled from the results of the Ref. [4] is shown in the inset. 
tal results reported in reference [4] to a $20 \mathrm{~m}$ distance from the source.

Close inspection of the obtained results (Fig. 3) shows the following. First, it is clear that the radiation field is not fully coherent but is rather partially coherent at a few hundred microns length scale. This was already expected from our mode analysis of the radiation field with FLASH parameters. However, a comparison with our previous calculations [see Fig. 1(d)] shows that the measured values of the degree of coherence for different slit separations are considerably lower than ultimately predicted by the model. The obtained values of the degree of coherence can be approximated by a Lorentzian function $L(x)=1 /[1+$ $\left.(x / \xi)^{2}\right]$ with parameters $\xi_{H}=300 \pm 15 \mu \mathrm{m}$ and $\xi_{V}=$ $250 \pm 13 \mu \mathrm{m}$ which can be considered as an estimate of the coherence length at that distance from the source. These values show that the coherence properties of the FEL are of the same order of magnitude in both vertical and horizontal directions, though slightly higher in the horizontal direction. The apparent source size corresponding to these values for the coherence lengths can be calculated using the same Gaussian Schell-model. The analysis gives a value $\sigma_{I}=180 \mu \mathrm{m}$ which is about 2.5 times larger than considered in our theoretical modelling. This size is in very good agreement with the source size observed with the wave front sensor at similar operating conditions of FLASH [25].

In summary we presented a theoretical and experimental analysis of the coherence properties of FLASH at a fundamental wavelength of $13.7 \mathrm{~nm}$. We used a very general statistical optics theory to model the mode structure and coherence properties of the beam. With this approach the spectral density distribution as well as the complex degree of coherence can be calculated at any distance from the source with just a few input parameters. This very general modelling can be applied as an effective and useful tool for describing the coherence properties of SASE FELs. This approach may also be conveniently extended in future to calculate the coherence properties of FEL beams passing through different optical elements [27,28]. Our analysis shows that FLASH is a highly coherent source which can be well described by a few coherent modes. We also performed a detailed analysis of the double-slit measurements at FLASH which showed that the values of the degree of coherence are similar for the horizontal and vertical directions, and the coherence lengths are about 300 microns at a distance $20 \mathrm{~m}$ downstream from the source.

We greatly appreciate the work of the scientific and technical team at FLASH, in particular, the machine operators and run coordinators. We acknowledge E. Weckert and $\mathrm{H}$. Zacharias for their support and A. Mancuso for a careful reading of the manuscript.
*Also at Physics Department, WWU Münster, WilhelmKlemm-Str. 9, D-48149 Münster, Germany.

Corresponding author. Ivan.Vartaniants@desy.de

[1] E. L. Saldin, E. A. Schneidmiller, and M. V. Yurkov, The Physics of Free Electron Lasers (Springer-Verlag, Berlin, 2000).

[2] J. Feldhaus et al., J. Phys. B 38, S799 (2005).

[3] J. Arthur et al., http://www-ssrl.slac.stanford.edu/LCLS/ CDR/; T. Tanaka and T. Shintake, http://www-xfel. spring8.or.jp/SCSSCDR.pdf; M. Altarelli et al., http:// xfel.desy.de/tdr/index_eng.html.

[4] W. Ackermann et al., Nat. Photon. 1, 336 (2007).

[5] J. Miao et al., Nature (London) 400, 342 (1999).

[6] M. A. Pfeifer et al., Nature (London) 442, 63 (2006).

[7] H. N. Chapman et al., Nature Phys. 2, 839 (2006).

[8] H.N. Chapman et al., Nature (London) 448, 676 (2007).

[9] I. A. Vartanyants et al., J. Synchrotron Radiat. 14, 453 (2007).

[10] R. Neutze et al., Nature (London) 406, 752 (2000).

[11] E. L. Saldin et al., Opt. Commun. 281, 1179 (2008).

[12] J.W. Goodman, Statistical Optics (Wiley, New York, 1985).

[13] L. Mandel and E. Wolf, Optical Coherence and Quantum Optics (Cambridge University Press, Cambridge, England, 1995).

[14] B. J. Thomson and E. Wolf, J. Opt. Soc. Am. 47, 895 (1957).

[15] C. Chang et al., Opt. Commun. 182, 25 (2000).

[16] D. Paterson et al., Opt. Commun. 195, 79 (2001).

[17] A. Tonomura et al., Am. J. Phys. 57, 117 (1989).

[18] M. Arndt et al., Nature (London) 401, 680 (1999).

[19] To keep equations as short as possible we will omit $\omega$ from our future equations.

[20] F. Gori, Opt. Commun. 34, 301 (1980).

[21] A. Starikov and E. Wolf, J. Opt. Soc. Am. 72, 923 (1982).

[22] Because of the symmetry of the Gaussian-Schell model in the transverse direction and decomposition of the transverse modes we will consider below only one transverse direction.

[23] F. Gori, Opt. Commun. 46, 149 (1983).

[24] When SASE FEL operates in the exponential growth regime it contains large intensity fluctuations and beam parameters can be substantially different from their saturation values. Measurements with a Hartman type wave front sensor [25] showed a source size up to 2.5 times larger for the exponential regime as compared with the saturated regime of operation.

[25] M. Kuhlmann et al., HASYLAB Annual Report 2006, edited by W. Caliebe, W. Drube, and J. R. Schneider, Part I, p. 213.

[26] The natural bandwidth of radiation at FLASH is on the order of $1 \%$ at the $13.7 \mathrm{~nm}$ wavelength.

[27] H. T. Yura and S. G. Hanson, J. Opt. Soc. Am. A 4, 1931 (1987).

[28] A. M. Zysk et al., Phys. Rev. Lett. 95, 043904 (2005). 